\title{
Cohort Profile: The Swiss Eosinophilic Esophagitis Cohort Study (SEECS)
}

\author{
Ekaterina Safroneeva ${ }^{a}$ Catherine Saner $^{\mathrm{b}}$ Jean-Benoît Rossel ${ }^{\mathrm{b}}$ \\ Delphine Golay $^{b}$ Valérie Pittet ${ }^{b}$ Sébastien Godat ${ }^{c}$ Stefan Diem ${ }^{d}$ \\ Patrick Aepli $^{d}$ Mikael Sawatzki $^{\text {e Jan Borovicka }}{ }^{e}$ Konstantin Burgmann ${ }^{f}$ \\ Pascal Juillerat ${ }^{\mathrm{g}} \quad$ Peter Netzer $^{\mathrm{h}} \quad$ Alexander Sendensky ${ }^{\mathrm{h}}$ Petr Hruz ${ }^{\mathrm{i}}$ \\ Marc Girardin j Luc Biedermann ${ }^{k}$ Thomas Greuterk Stephan Vavrickak \\ Pierre Michettic, ${ }^{c}$ Christoph Mueller ${ }^{m}$ Alex Straumann ${ }^{k, n}$ \\ Alain M. Schoepfer ${ }^{c}$ on behalf of the Swiss EoE Cohort Study Group
}

\begin{abstract}
${ }^{a}$ Institute of Social and Preventive Medicine, University of Bern, Bern, Switzerland; ${ }^{b}$ Institute of Social and Preventive Medicine, Centre Hospitalier Universitaire Vaudois and University of Lausanne, Lausanne, Switzerland; ' Division of Gastroenterology and Hepatology, Centre Hospitalier Universitaire Vaudois, Lausanne, Switzerland; ${ }^{\mathrm{d}}$ Division of Gastroenterology and Hepatology, Kantonsspital Luzern, Luzern, Switzerland; ' Division of Gastroenterology

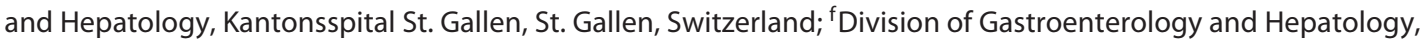
Kantonsspital Fribourg, Fribourg, Switzerland; ${ }^{9}$ Division of Gastroenterology and Hepatology, Inselspital and University of Bern, Bern, Switzerland; h GastroZentrum Netzer AG, Lindenhofspital, Bern, Switzerland; 'Division of Gastroenterology and Hepatology, University Hospital Basel, Basel, Switzerland; ' Division of Gastroenterology and Hepatology, University Hospital of Geneva, Geneva, Switzerland; ' Division of Gastroenterology and Hepatology, University Hospital Zurich, Zurich, Switzerland; 'Crohn's and Colitis Center, La Source, Lausanne, Switzerland; mDivision of Experimental Pathology, Institute of Pathology, University of Bern, Bern, Switzerland; nSwiss EoE Clinic, Olten, Switzerland
\end{abstract}

\section{Keywords \\ Cohort profile - Cohort study - Eosinophilic esophagitis . Gastroesophageal reflux disease · Proton pump inhibitor-responsive esophageal eosinophilia}

\footnotetext{
Abstract

Background and Aims: The prospective, observational Swiss Eosinophilic Esophagitis Cohort Study (SEECS) was set up in 2015 with the following goals in mind: (1) to provide up-todate epidemiologic data; (2) to assess the appropriateness of care; (3) to evaluate the psychosocial impact; and (4) to foster
}

translational research projects. Data capture relies on validated instruments to assess disease activity and focuses on epidemiologic variables and biosamples (esophageal biopsies and blood specimens). An annual inclusion of 70 new patients with eosinophilic esophagitis (EoE) or proton pump inhibitor-responsive esophageal eosinophilia (PPI-REE) is intended. We herein describe the SEECS cohort profile. Methods: The SEECS includes adult patients (age $\geq 18$ years) with EoE or PPI-REE diagnosed according to published criteria. After inclusion, the patients are typically seen once a year for a clinical and endoscopic/histologic follow-up examination. Data are captured using validated questionnaires. Biosam-

\section{KARGER}

(c) 2018 S. Karger AG, Basel

E-Mail karger@karger.com

www.karger.com/iid
Alain M. Schoepfer, MD, PD + MERClin

Division of Gastroenterology and Hepatology

Centre Hospitalier Universitaire Vaudois/CHUV

Rue de Bugnon 44, 07/2425, CH-1011 Lausanne (Switzerland)

E-Mail alain.schoepfer@chuv.ch 
ples from patients with gastroesophageal reflux disease (GERD) and controls with a healthy esophagus are collected as well. Results: From January 2016 to July 2017, a total of 111 patients with EoE and 10 patients with PPI-REE were recruited. In addition, esophageal biopsies and blood samples from 11 patients with GERD and 20 controls with a healthy esophagus were collected. The mean age of the patients with EoE and those with PPI-REE was $39.6 \pm 12.9$ and $44.6 \pm$ 15.6 years, respectively. A male predominance was found among both the patients with EoE (77.5\%) and those with PPI-REE (70\%). Concomitant allergic disorders were found in $79.3 \%$ of the patients with $\mathrm{EoE}$ and $90 \%$ of the patients with PPI-REE. At inclusion, the EoE patients were treated with the following therapeutic regimens: no therapy $(0.9 \%)$, PPI $(36 \%)$, swallowed topical corticosteroids (82.9\%), elimination diets (15.3\%), and esophageal dilation (19.8\%). Conclusions: The SEECS is the first national cohort study of patients with EoE or PPI-REE. The SEECS will provide up-to-date epidemiologic data and foster translational research projects.

(c) 2018 S. Karger AG, Basel

\section{Introduction}

\section{Key Points on Eosinophilic Esophagitis}

The first consecutive case series of patients with eosinophilic esophagitis (EoE) were published in 1993 and 1994 by Attwood et al. [1] and Straumann et al. [2] in the USA and in Switzerland, respectively. EoE is currently defined as "a chronic, immune/antigen-mediated, esophageal disease, characterized clinically by symptoms related to esophageal dysfunction and histologically by eosinophil-predominant inflammation" [3, 4]. EoE patients are mostly male, and a high prevalence of allergies against inhalable or food allergens is observed [5-7]. Symptoms, mostly dysphagia, have typically already been present for years before EoE is diagnosed [8]. The disease is observed in industrialized countries with increasing frequency. The current incidence ranges from 1 to 20 new patients per 100,000 inhabitants per year (mean value 7 ) and the prevalence ranges between 13 and 49 cases per 100,000 inhabitants [4].

In recent years, clinicians and researchers have become aware of the fact that some patients with EoE-typical clinical, endoscopic, and histologic features responded to proton pump inhibitors (PPI). Of note, these patients did not suffer from gastroesophageal reflux disease (GERD). Thus, the term "proton pump inhibitor-responsive esophageal eosinophilia" (PPI-REE) was created [4, 5]. Patients with PPI-REE cannot be discriminated from "real" EoE patients based on clinical, genetic, endoscopic, and histologic features, which suggests that these patients are within the spectrum of EoE and do not represent a separate disease entity $[9,10]$.

The pathogenesis of EoE (and PPI-REE) is still incompletely understood [6]. It is generally accepted that EoE results from a complex interplay between genetic, environmental, and host immune systemic factors, comparable to inflammatory bowel disease (IBD) [6]. There exists increasing evidence that EoE is a chronic progressive disease that - if eosinophil-predominant inflammation remains untreated - can lead to esophageal remodeling processes with fibrous tissue deposition and consecutive stricture formation [11-15]. The presence of esophageal strictures represents the key risk factor for the feared food bolus impactions that might necessitate endoscopic disimpaction as an emergency procedure $[16,17]$.

There exist several reasons for treating active EoE: first, to reduce EoE-related symptoms and to improve EoE-related quality of life [18, 19], and second, in the long run, to reduce or prevent esophageal remodeling processes that are associated with stricture formation and food bolus impaction. The therapy options for treating EoE can be summarized as the " $3 D$ 's, " which stands for "drugs, diet, and dilation." For an in-depth review of the therapy recommendations regarding the use of elimination diets, drug therapy, and esophageal dilation, we kindly refer to evidence-based statements and recommendations for diagnosis and management in children and adults [4].

\section{Recent Achievements in Standardizing the Assessment} of Disease Activity in EoE

Disease activity in EoE can be assessed by patient-reported outcomes (PRO) and clinician-reported outcomes (ClinRO) [20, 21]. Concerted efforts of EoE researchers have led to the development and validation of EoE-specific instruments for measuring clinical, endoscopic, and histologic EoE activity. EoE-specific symptoms are assessed by the Eosinophilic Esophagitis Activity Index (EEsAI) PRO instrument or the Dysphagia Symptom Questionnaire (DSQ) $[22,23]$. The development and validation of the EEsAI PRO instrument followed recommendations from regulatory authorities [24]. EoE-specific quality of life can be measured using the validated score developed by Taft et al. [18]. Endoscopic activity is assessed using the EREFS (Endoscopic Reference Score) classification and grading system, which evaluates exudates, rings, edema, furrows, and strictures [25]. Histologic activity has been evaluated by counting the peak eosinophil number per high-power field (400-fold magni- 
fication). Recently, a histologic score has been published that contains 8 distinct items for the assessment of EoE activity [26]. In EoE, both PRO and ClinRO measures contribute to the assessment of disease activity [21]. As such, most clinical trials assess both PRO and ClinRO (e.g., histologic and endoscopic) to obtain a global view of EoE activity. As yet, there exists no pharmacologic treatment specifically for EoE that has been granted approval from regulatory authorities.

\section{Rationale for the Study}

With the first patients described in 1993, EoE is considered to be a "young" disease with many unknowns. A prospective cohort study of EoE and PPI-REE patients is best suited to address some of the unmet needs that have recently been summarized in the updated 2017 EoE guidelines [4]. Topics to be addressed include, among others, the following questions: what environmental risk factors can be identified as being associated with EoE and PPI-REE? Why do antigens that have been present in staple foods for centuries cause EoE now? Is treatment with swallowed topical steroids and PPI more effective than monotherapy with swallowed topical steroids? What factors determine if an EoE patient will rapidly develop a stricturing phenotype? What is an optimal histologic cutoff (peak eosinophils $/ \mathrm{mm}^{2}$ ) to be reached in the long run to prevent stricture formation? Are there safety issues with patients on PPI (e.g., osteoporosis) or on topical steroids swallowed over a long period of time (e.g., adrenal suppression)?

As such, the general goal of the Swiss Eosinophilic Esophagitis Cohort Study (SEECS) is to provide the scientific community with a disease-oriented prospective cohort of patients suffering from EoE and PPI-REE. The SEECS will (1) provide up-to-date epidemiologic data (e.g., regarding the clinical course of EoE) that allow investigating risk factors associated with a favorable or unfavorable disease course; (2) document medical care given to patients in order to assess the appropriateness of care; (3) evaluate the psychosocial impact of EoE; and (4) foster translational research projects making use of the biobank (e.g., evaluation of esophageal barrier dysfunction).

\section{Cohort Description}

\section{Pilot Study}

To learn more about the incidence and prevalence of EoE in Switzerland, our research group conducted population-based studies in two indicator regions of Switzerland. The first one was conducted in Olten county in the German-speaking part of Swit- zerland, and the other one in the canton of Vaud in the Frenchspeaking part of Switzerland $[27,28]$. In Olten county, we found an EoE prevalence of about 1 in 2,000 inhabitants (in the period of 2007-2009), and in the canton of Vaud of 1 in 4,000 inhabitants (in the year 2013). While in Olten county EoE was diagnosed for the first time already in 1989, in the canton of Vaud it was diagnosed only in 2003. The EoE incidence rates were similar between Olten county (7.4/100,000 in the period between 2007 and 2009) and the canton of Vaud (6.3/100,000 in the year 2013). Thus, we are anticipating that the EoE prevalence in the French-speaking part of Switzerland will be on the rise in the near future, and probably approach prevalence rates similar to those in the Germanspeaking part of Switzerland in due time.

\section{Setting, Location, and Relevant Data}

The SEECS datacenter is located at the Institute of Social and Preventive Medicine of the University of Lausanne in Switzerland. The SEECS datacenter is incorporated into the Swiss IBD Cohort Study (SIBDCS), which has been recruiting patients with IBD since 2006 [29]. The SEECS became part of the SIBDCS as a large nested project in 2015. The reasons for initiating the SEECS were the observation of a rapidly increasing incidence and prevalence of EoE and PPI-REE in Switzerland and the realization that there are several similarities between EoE and IBD [30]. The core team consists of a study manager (C.S.), a questionnaire designer (E.S.), a data manager (D.G.), a biostatistician (J.-B.R.), a study center supervisor (V.P.), and the principal investigator (A.M.S.).

The SEECS project started in 2015 with the creation of questionnaires for screening, enrollment, and follow-up visits. Online supplementary Table 1 (for all online suppl. material, see www. karger.com/doi/10.1159/000486131) gives an overview of the questionnaires and the persons responsible for their completion. The questionnaires for physicians/study nurses were created in English, whereas the patient questionnaires were created in German, French, and English.

In addition, documents for the institutional review boards (IRB) were written. Switzerland has 7 ethics committees which cover a total of 26 cantons (country districts). Figure 1 shows the location of the major centers of inclusion and their working areas. The SEECS project was submitted to the ethics committee of the canton of Vaud (CER-VD), which acts as the leading committee. After approval of the project by the CER-VD in 2015 (protocol No. 148/15), the SEECS documents were sent to 5 other IRB (EKNZ, Bern, Geneva, EKOS, and Zurich), which granted approval in 2015-2016. These 6 IRB cover the entire German- and Frenchspeaking parts of Switzerland. The documents have not yet been submitted to the IRB of the canton of Ticino for the inclusion of Italian-speaking patients. SIBDCS nurses at IBD centers also include EoE patients. Patient inclusion started in January 2016. Although being a purely observational study, the SEECS was rated as category "B" by the CER-VD, which means that there is a minimal risk for potential harm due to acquisition of blood samples and esophageal biopsies for study purposes. As such, all included patients are covered by an insurance policy that has been issued by the Centre Hospitalier Universitaire Vaudois and which is valid for all participating patients irrespective of the location of inclusion.

\section{Eligibility Criteria and Recruitment Process}

The eligibility of adult patients (age $\geq 18$ years) is assessed by use of the screening questionnaire. For inclusion, patients must 


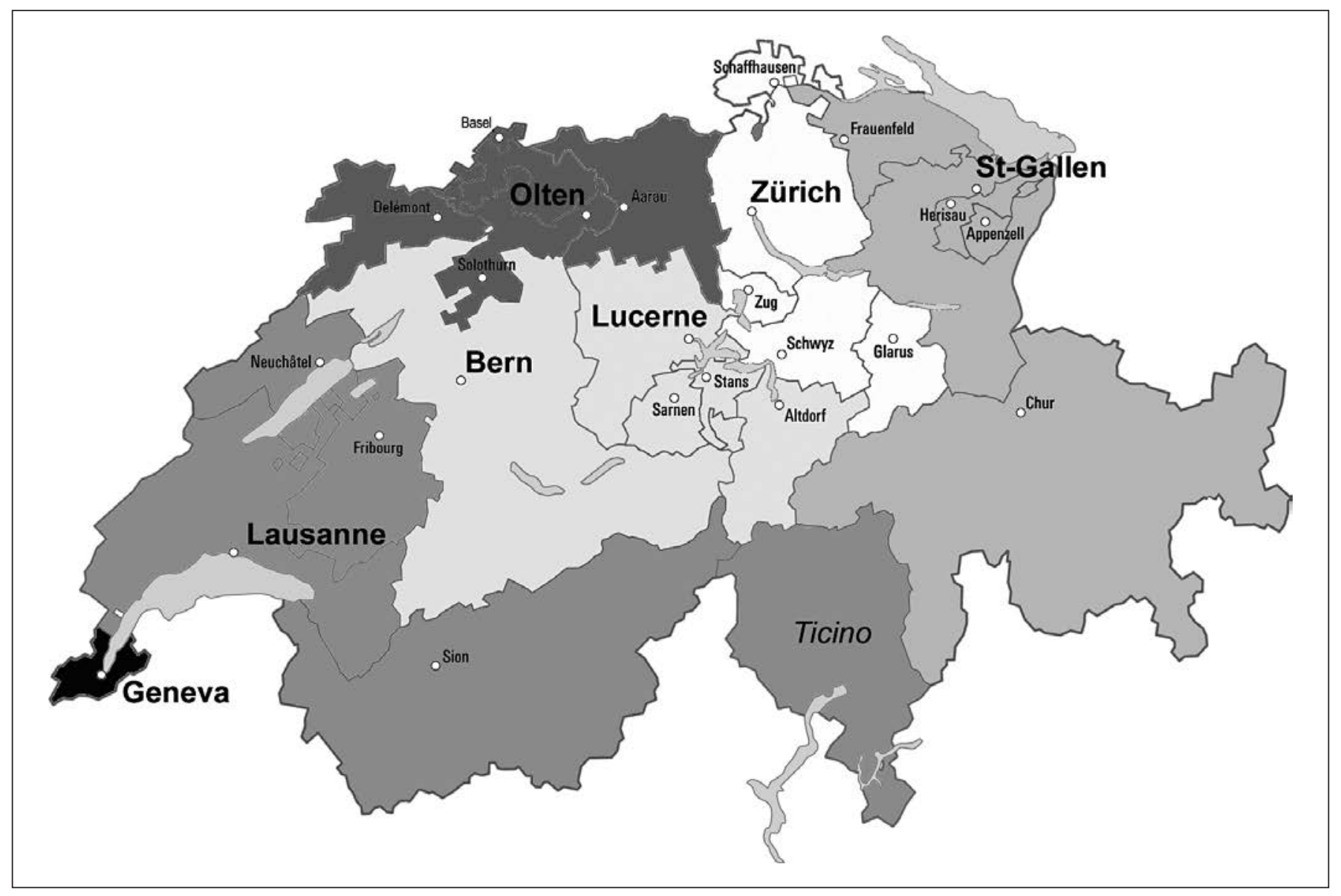

Fig. 1. Location of the major centers of inclusion and their working areas.

fulfil the following criteria according to the diagnostic guidelines: (1) symptoms of esophageal dysfunction and (2) a peak eosinophil count $\geq 15$ per high-power field (400× magnification) despite treatment with PPI (single or double standard dose) for $\geq 8$ weeks [5]. Patients with PPI-REE must fulfil the following criteria for inclusion: (1) symptoms of esophageal dysfunction; (2) clinical and histologic response upon 8-week treatment with PPI in single or double standard dose; and (3) no GERD [5]. Patients with EoE and concomitant GERD can be included if the diagnosis of EoE and GERD has been established based on accepted diagnostic criteria [5].

Patients are excluded in case of a permanent address outside Switzerland, refusal to sign the informed consent form, and/or conditions other than EoE or PPI-REE associated with esophageal eosinophilia, such as eosinophilic gastroenteritis, esophageal Crohn disease, connective tissue disorder, esophageal infection, etc. [5].

The SEECS datacenter hands out sets of paper-based questionnaires to gastroenterologists willing to recruit patients. The questionnaires are labeled with stickers that have a unique 9-digit identifier. The first 2 digits identify the recruiting center, whereas digits 3-5 identify the recruiting physician, and digits 6-9 encode the patient. Patients first read the SEECS information sheet and pro- vide their consent in case of willingness to participate. Additional consent is provided if the patient agrees with the performance of genetic studies on with his/her blood samples and esophageal biopsies. The patients and the including gastroenterologist sign the informed consent sheet 3 times ( 1 sheet for the patient, 1 sheet for the datacenter, and 1 sheet for the patient file). The patients then sign a sheet that provides their contact information; in addition they choose whether they agree to be contacted for the administration of additional questionnaires during the year. All questionnaires are then labeled with the unique identifier tags. Thereby, patient recruitment is anonymized. The identification key is stored at the datacenter (IUMSP Lausanne).

Patients are typically included during a scheduled follow-up esophagogastroduodenoscopy. They complete the PRO questionnaire regarding symptoms and EoE-specific quality of life before the upper endoscopy. Symptoms are assessed using the validated EEsAI PRO questionnaire [22]. If willing, the patients also provide a blood sample for study purposes. They then undergo upper endoscopy with biopsy sampling for standard histology, which typically involves 3 biopsies from the distal and 3 biopsies from the proximal esophagus. These samples are fixed in formalin and sent to the local pathology institute in order to determine the severity of inflammatory and fibrotic features. In addition, 2 additional bi- 
Table 1. Variables captured by the SEECS questionnaires as well as the frequency of their completion

\begin{tabular}{|c|c|c|c|c|c|}
\hline Measurements & \multicolumn{3}{|c|}{ Questionnaire } & \multicolumn{2}{|c|}{ Frequency of data collection } \\
\hline Diagnosis & $\mathrm{x}$ & & $\mathrm{x}$ & $\mathrm{x}$ & $\mathrm{x}$ \\
\hline Clinical activity & $\mathrm{x}$ & $\mathrm{x}$ & & $\mathrm{x}$ & $\mathrm{x}$ \\
\hline Quality of life & & $\mathrm{x}$ & & $\mathrm{x}$ & $\mathrm{x}$ \\
\hline Histologic activity in EoE/PPI-REE & $\mathrm{x}$ & & & $\mathrm{x}$ & $\mathrm{x}$ \\
\hline Blood eosinophilia & $\mathrm{x}$ & & & $\mathrm{x}$ & $\mathrm{x}$ \\
\hline Current therapy for EoE/PPI-REE & $\mathrm{x}$ & & & $\mathrm{x}$ & $\mathrm{x}$ \\
\hline Current therapy for other conditions ${ }^{1}$ & $\mathrm{x}$ & & & $\mathrm{x}$ & $\mathrm{x}$ \\
\hline Disease activity & $\mathrm{x}$ & & & $\mathrm{x}$ & $\mathrm{x}$ \\
\hline Management decision & $\mathrm{x}$ & & & $\mathrm{x}$ & $\mathrm{x}$ \\
\hline Adverse events related to EoE drugs & $\mathrm{x}$ & & & & $\mathrm{x}$ \\
\hline New medical examinations & $\mathrm{x}$ & & & & $\mathrm{x}$ \\
\hline Complications & $\mathrm{x}$ & & & & $\mathrm{x}$ \\
\hline
\end{tabular}

SEECS, Swiss Eosinophilic Esophagitis Cohort Study; EoE, eosinophilic esophagitis; PPI-REE, proton pump inhibitor-responsive esophageal eosinophilia; GERD, gastroesophageal reflux disease. ${ }^{1}$ Includes therapies for concomitant asthma, rhinoconjunctivitis, eczema, or GERD.

opsies each from the distal and the proximal esophagus are taken for study purposes. These samples are stored in RNAlater (Invitrogen) to stabilize cellular RNA and DNA. The blood samples and esophageal biopsies are sent by mail to the biobank at the Institute of Pathology of the University of Bern. Besides biosamples of patients with EoE and PPI-REE, biosamples of patients with GERD and controls with a healthy esophagus are collected as well, and are stored in the central biobank. Inclusion of these control samples will facilitate translational studies, e.g., regarding esophageal barrier dysfunctions, and thus elucidate disease pathogenesis.

The including gastroenterologist completes the physician questionnaires after endoscopy using standardized instruments to assess endoscopic and histologic activity $[25,26]$. The patients are typically seen once a year for an endoscopic and histologic followup examination, during which the follow-up questionnaires are administered. Table 1 provides an overview of the variables collected, as well as the frequency of data collection. The follow-up questionnaire can also be used for an unscheduled event such as a medical visit due to acute food bolus impaction that necessitates endoscopic removal.

\section{Findings to Date}

In January 2016, patient recruitment started in the 5 university hospitals of Lausanne, Geneva, Basel, Bern, and Zurich, as well as in the hospitals of Lucerne, St. Gal-

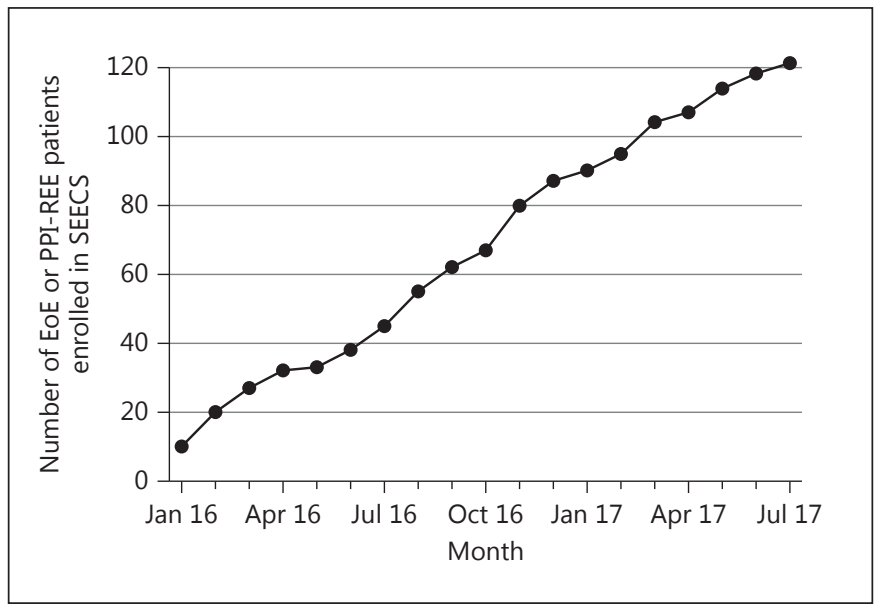

Fig. 2. Cumulative numbers of patients with eosinophilic esophagitis (EoE) and proton pump inhibitor-responsive esophageal eosinophilia (PPI-REE) included between January 2016 and July 2017. SEECS, Swiss Eosinophilic Esophagitis Cohort Study.

len, and the Swiss EoE Clinic in Olten, Switzerland. The project aims for a yearly recruitment rate of 70 new patients with EoE or PPI-REE. Figure 2 shows the number of patients with EoE or PPI-REE recruited per month 
Table 2. Baseline characteristics of the included subjects as well as numbers of biosamples as of July 2017

\begin{tabular}{|c|c|c|c|c|}
\hline & $\mathrm{EoE}$ & PPI-REE & GERD & $\begin{array}{l}\text { Healthy } \\
\text { controls }\end{array}$ \\
\hline Subjects & 111 & 10 & 11 & 20 \\
\hline Male/female & $86 / 25$ & $7 / 3$ & $4 / 7$ & $7 / 13$ \\
\hline Mean age at inclusion $\pm S D$, years & $39.6 \pm 12.9$ & $44.6 \pm 15.6$ & $59.9 \pm 12.7$ & $51.1 \pm 15.2$ \\
\hline Mean diagnostic delay \pm SD, years & $4.3 \pm 3.8$ & $2.6 \pm 3.0$ & - & - \\
\hline Concomitant GERD & $18(16.2 \%)$ & - & - & - \\
\hline \multicolumn{5}{|l|}{ Concomitant allergies } \\
\hline None & $23(20.7 \%)$ & $1(10 \%)$ & - & - \\
\hline Overall & $82(73.9 \%)$ & $7(70 \%)$ & - & - \\
\hline Rhinoconjunctivitis & $57(51.4 \%)$ & $6(60 \%)$ & - & - \\
\hline Asthma & $35(31.5 \%)$ & $4(40 \%)$ & - & - \\
\hline Neurodermatitis & $11(9.9 \%)$ & $3(30 \%)$ & - & - \\
\hline Food allergies & $39(35.1 \%)$ & $5(50 \%)$ & - & - \\
\hline Missing information & $6(5.4 \%)$ & $2(20 \%)$ & - & - \\
\hline \multicolumn{5}{|l|}{ Current therapy } \\
\hline None & $1(0.9 \%)$ & 0 & 10 & - \\
\hline Any & $107(96.4 \%)$ & $10(100 \%)$ & 0 & - \\
\hline PPI & $40(36.0 \%)$ & $9(90 \%)$ & 0 & - \\
\hline Swallowed topical corticosteroids & $92(82.9 \%)$ & 0 & 0 & - \\
\hline Elimination diet & $17(15.3 \%)$ & $2(20 \%)$ & 0 & - \\
\hline Esophageal dilation & $22(19.8 \%)$ & $2(20 \%)$ & 0 & - \\
\hline Missing information & $3(2.7 \%)$ & 0 & $1(9.1 \%)$ & - \\
\hline Subjects providing esophageal biopsies ${ }^{1}$ & 147 & 12 & 15 & 19 \\
\hline \multicolumn{5}{|l|}{ Subjects providing blood samples } \\
\hline EDTA & 148 & 10 & 16 & 16 \\
\hline Serum & 77 & 5 & 8 & 8 \\
\hline
\end{tabular}

EoE, eosinophilic esophagitis; PPI-REE, proton pump inhibitor-responsive esophageal eosinophilia; GERD, gastroesophageal reflux disease. ${ }^{1}$ Regarding biosamples: 1 patient could be counted several times if $>1$ endoscopy was performed.

since January 2016. By the end of July 2017, a total of 111 patients with EoE and 10 patients with PPI-REE were recruited. A total of $5 \mathrm{EoE}$ patients $(5 / 126 ; 4 \%)$ declared their unwillingness to participate. Thus, the participation rate of patients with EoE and PPI-REE was 96\%. In addition, biopsies and blood samples from 11 patients with GERD and 20 controls with a healthy esophagus were collected. The baseline characteristics of the patients and the numbers of biosamples as of July 2017 are shown in Table 2. The mean age of the patients with EoE was $39.6 \pm 12.9$ years, and that of the PPI-REE patients was $44.6 \pm 15.6$ years. A male predominance was found for both the patients with EoE (77.5\%) and those with PPI-REE (70\%). Concomitant allergic disorders were found in $79.3 \%$ of the patients with EoE and $90 \%$ of the patients with PPIREE. Nearly all patients (99.1\%) with EoE had a distinctive therapy at inclusion, with swallowed topical steroids
(82.9\%) being the most frequently applied drug, followed by PPI (36\%). Elimination diets were practiced by $15.3 \%$ of the EoE patients at inclusion. All patients with PPIREE were on treatment at inclusion, with PPI being prescribed to $90 \%$ of these patients.

\section{Strengths and Limitations}

The SEECS has several strengths and also some limitations. To the best of our knowledge, it provides the first national cohort of patients with EoE and PPI-REE. Patients are included based on strict diagnostic criteria [4]. Assessment of clinical and biologic disease activity is based on validated instruments designed specifically for EoE and PPI-REE [22, 25, 26]. The cross-sectional capture of data regarding clinical activity, EoE-specific quality of life, and endoscopic and histologic activity will help to clarify the controversially discussed relationship between 
PRO and biologic activity [31]. Follow-up information is typically captured once a year. Biosamples are collected not only from patients with EoE and PPI-REE, but also from patients with GERD and from controls with a healthy esophagus, which is critical for translational projects.

As a first limitation, the SEECS is not population based. In addition, we currently abstain from the inclusion of pediatric patients with EoE and PPI-REE. A validated PRO instrument in German and French for pediatric EoE patients is currently lacking, which hampers the inclusion of pediatric patients. The EEsAI study group is currently developing such an instrument. Hence, the inclusion of pediatric EoE patients has been designated for a later stage. Second, for the time being, we have not created any questionnaires for the Italian-speaking canton of Ticino in southern Switzerland. As of the end of 2016, the canton of Ticino had 354,357 inhabitants, which corresponds to $4.43 \%$ of the entire Swiss population (currently about 8 million people). The inclusion of Italian-speaking patients is planned in a later step.

\section{Acknowledgments}

The authors thank all members of the SEECS who were involved in the acquisition of data: Claudia Anderegg; Patrick Aepli; Peter Bauerfeind; Christoph Beglinger; Stefan Begré; Dominique Belli; José M. Bengoa; Luc Biedermann; Beat Bigler; Janek Binek; Mirjam Blattmann; Stephan Boehm; Jan Borovicka; Christian P. Braegger; Nora Brunner; Patrick Bühr; Emanuel Burri; Sophie Buyse; Matthias Cremer; Dominique H. Criblez; Philippe de Saussure; Lukas Degen; Joakim Delarive; Christopher Doerig; Barbara Dora; Mara Egger; Tobias Ehmann; Ali El-Wafa; Matthias Engelmann; Jessica Ezri; Christian Felley; Markus Fliegner; Nicolas Fournier; Pascal Frei; Remus Frei; Michael Fried; Florian Froehlich; Christian Funk; Raoul Ivano Furlano; Suzanne Gallot-Lavallée; Martin Geyer; Marc Girardin; Delphine Golay; Tanja Grandinetti; Beat Gysi; Horst Haack; Johannes Haarer; Beat Helbling; Peter Hengstler; Denise Herzog; Cyrill Hess; Klaas Heyland; Thomas Hinterleitner; Philippe Hiroz; Claudia Hirschi; Petr Hruz; Rika Iwata; Res Jost; Pascal Juillerat; Vera Kessler Brondolo; Christina Knellwolf; Christoph Knoblauch; Henrik Köhler; Rebekka Koller; Claudia Krieger-Grübel; Gerd Kullak-Ublick; Patrizia Künzler; Markus Landolt; Rupprecht Lange; Frank Serge Lehmann; Andrew Macpherson; Philippe Maerten; Michel H. Maillard; Christine Manser; Michael Manz; Urs Marbet; George Marx; Christoph Matter; Valérie McLin; Rémy Meier; Martina Mendanova; Christa Meyenberger; Pierre Michetti; Benjamin Misselwitz; Bernhard Morell; Patrick Mosler; Christian Mottet; Christoph Müller; Pascal Müller; Beat Müllhaupt; Claudia Münger-Beyeler; Leila Musso; Andreas Nagy; Michaela Neagu; Cristina Nichita; Jan Niess; Natacha Noël; Andreas Nydegger; Nicole Obialo; Carl Oneta; Cassandra Oropesa; Ueli Peter; Daniel Peternac; Laetitia Marie Petit; Franziska Piccoli-Gfeller; Julia Beatrice Pilz; Valérie Pittet; Nadia Raschle; Ronald Rentsch; Sophie Restellini; Jean-Pierre Richterich;
Sylvia Rihs; Marc Alain Ritz; Jocelyn Roduit; Daniela Rogler; Gerhard Rogler; Jean-Benoitt Rossel; Markus Sagmeister; Gaby Saner; Bernhard Sauter; Mikael Sawatzki; Michela Schäppi; Michael Scharl; Martin Schelling; Susanne Schibli; Hugo Schlauri; Sybille Schmid Uebelhart; Jean-François Schnegg; Alain Schoepfer; Frank Seibold; Mariam Seirafi; Gian-Marco Semadeni; David Semela; Arne Senning; Marc Sidler; Christiane Sokollik; Johannes Spalinger; Holger Spangenberger; Philippe Stadler; Michael Steuerwald; Alex Straumann; Bigna Straumann-Funk; Michael Sulz; Joël Thorens; Sarah Tiedemann; Stephan Vavricka; Francesco Viani; Jürg Vögtlin; Roland Von Känel; Alain Vonlaufen; Dominique Vouillamoz; Rachel Vulliamy; Jürg Wermuth; Helene Werner; Paul Wiesel; Reiner Wiest; Tina Wylie; Jonas Zeitz; Dorothee Zimmermann.

\section{Collaboration}

All data are centralized in the Institute of Social and Preventive Medicine of the University of Lausanne, Lausanne, Switzerland. The SEECS is part of the SIBDCS. All logistic aspects of the data flow are managed by the datacenter, including data control and statistical analyses. Gastroenterologists who include patients in the SEECS receive an individual feedback sheet and an update on the number of patients included once a year. It is planned that the global results of the SEECS will be accessible on the website of the SIBDCS (http://www.ibdcohort.ch). The manual listing the standard operating procedures for submission and evaluation of the projects of the SIBDCS applies also to the SEECS. These standard operating procedures were prepared by the scientific committee of the SIBDCS and have been in place since 2006. Every researcher interested in collaborating with the SEECS can submit a standardized proposal for a scientific project. The template for submission of a scientific project is available on http://www.ibdcohort.ch. All submitted projects are peer-reviewed and subject to a final decision by the scientific committee.

\section{Statement of Ethics}

This research project is conducted in full compliance with research ethics norms. The SEECS project has undergone evaluation and approval of all Swiss IRBs with the exception of the Italianspeaking canton of Ticino, where the project has not yet been submitted. All study patients participate in the SEECS on a voluntary basis. Patients are included exclusively upon signing informed consent forms. The confidentiality and anonymity of the research respondents is respected based on strict data management rules that have been in place for the Swiss IBD Cohort Study since 2006.

\section{Disclosure Statement}

None of the authors have any conflicts of interest to disclose.

\section{Funding Sources}

The SEECS is supported by grants from the Swiss National Science Foundation (SNSF 33CS30_148422 [Gerhard Rogler] and 32473B_160115 [Alain M. Schoepfer]). 


\section{Author Contributions}

1 = study concept and design; 2 = acquisition of data; $3=$ analysis and interpretation of data; $4=$ drafting of the manuscript; $5=$ critical revision of the manuscript for important intellectual content; $6=$ statistical analysis; $7=$ technical or material support; $8=$ study supervision.
E. Safroneeva: 1-6; C. Saner: 1-5 and 7; J.-B. Rossel: 1-6; D. Golay: $2-5$ and 7; V. Pittet: $2-5$; S. Godat: $1-5$; S. Diem: $2-5$ and 7; P. Aepli: $2-5$ and 7; M. Sawatzki: $2-5$ and 7; J. Borovicka: $2-5$ and 7; K. Burgmann: 2-5 and 7; P. Juillerat: $2-5$ and 7; P. Netzer: 2-5 and 7; A. Sendensky: $2-5$ and 7; P. Hruz: $2-5$ and 7; M. Girardin: 2-5 and 7; L. Biedermann: 2-5 and 7; T. Greuter: 2-5 and 7; S. Vavricka: 2-5 and 7; P. Michetti: 2-5 and 7; C. Mueller: 2-5 and 7; A. Straumann: 2-5 and 7; A.M. Schoepfer: 1-8.

\section{References}

1 Attwood SE, Smyrk TC, Demeester TR, Jones JB: Esophageal eosinophilia with dysphagia, a distinct clinicopathologic syndrome. Dig Dis Sci 1993;38:109-116.

2 Straumann A, Spichtin HP, Bernoulli R, et al: Idiopathic eosinophilic esophagitis: a frequently overlooked disease with typical clinical aspects and discrete endoscopic findings (in German with English abstract). Schweiz Med Wochenschr 1994;124:1419-1429.

3 Liacouras CA, Furuta GT, Hirano I, et al: Eosinophilic esophagitis: updated consensus recommendations for children and adults. J Allergy Clin Immunol 2011;128:3-20.

4 Lucendo AJ, Molina-Infante J, Arias Á, et al: Guidelines on eosinophilic esophagitis: evidence-based statements and recommendations for diagnosis and management in children and adults. United European Gastroenterol J 2017;5:335-358.

5 Dellon ES, Gonsalves N, Hirano I, et al: ACG clinical guideline: evidenced based approach to the diagnosis and management of esophageal eosinophilia and eosinophilic esophagitis (EoE). Am J Gastroenterol 2013;108:679-692.

6 Furuta GT, Katzka DA: Eosinophilic esophagitis. N Engl J Med 2015;373:1640-1648.

7 Spergel JM, Brown-Whitehorn T, Beausoleil $\mathrm{JL}$, et al: Predictive values for skin prick test and atopy patch test for eosinophilic esophagitis. J Allergy Clin Immunol 2007;119:509511.

8 Straumann A, Spichtin HP, Grize L, et al: Natural history of primary eosinophilic esophagitis: a follow-up of 30 adult patients for up to 11.5 years. Gastroenterology 2003;125:16601669.

9 Moawad FJ, Schoepfer AM, Safroneeva E, et al: Eosinophilic oesophagitis and proton pump inhibitor-responsive oesophageal eosinophilia have similar clinical, endoscopic and histological findings. Aliment Pharmacol Ther 2014;39:603-608.

10 Molina-Infante J, Bredenoord AJ, Chang E, et al: Proton pump inhibitor-responsive oesophageal eosinophilia: an entity challenging current diagnostic criteria for eosinophilic oesophagitis. Gut 2016;65:524-531.
11 Assa'ad AH, Putnam PE, Collins $\mathrm{MH}$, et al: Pediatric patients with eosinophilic esophagitis: an 8-year follow-up. J Allergy Clin Immunol 2007;119:731-738.

12 Helou EF, Simonson J, Arora AS: 3-yr-followup of topical corticosteroid treatment for eosinophilic esophagitis in adults. Am J Gastroenterol 2008;103:2194-2199.

13 Spergel JM, Brown-Whitehorn TF, Beausoleil JL, et al: 14 years of eosinophilic esophagitis: clinical features and prognosis. J Pediatr Gastroenterol Nutr 2009;48:30-36.

14 Kagalwalla AF, Akhtar N, Woodruff SA, et al: Eosinophilic esophagitis: epithelial mesenchymal transition contributes to esophageal remodeling and reverses with treatment. J Allergy Clin Immunol 2012;129:1387-1396.

15 Schoepfer AM, Safroneeva E, Bussmann C, et al: Delay in diagnosis of eosinophilic esophagitis increases risk for stricture formation in a time-dependent manner. Gastroenterology 2013;145:1230-1236.

16 Lipka S, Kumar A, Richter JE: Impact of diagnostic delay and other risk factors on eosinophilic esophagitis phenotype and esophageal diameter. J Clin Gastroenterol 2016;50:134140.

17 Dellon ES, Kim HP, Sperry SL, et al: A phenotypic analysis shows that eosinophilic esophagitis is a progressive fibrostenotic disease. Gastrointest Endosc 2014;79:577-585.

18 Taft TH, Kern E, Kwiatek MA, et al: The Adult Eosinophilic Oesophagitis Quality of Life questionnaire: a new measure of healthrelated quality of life. Aliment Pharmacol Ther 2011;34:790-798.

19 Safroneeva E, Coslovsky M, Kuehni CE, et al: Eosinophilic oesophagitis: relationship of quality of life with clinical, endoscopic and histological activity. Aliment Pharmacol Ther 2015;42:1000-1010.

20 Schoepfer AM, Hirano I, Katzka DA: Eosinophilic esophagitis: overview of clinical management. Gastroenterol Clin North Am 2014; 43:329-344.

21 Schoepfer A, Safroneeva E, Straumann A: How to measure disease activity in eosinophilic esophagitis. Dis Esophagus 2016;29: 959-966.
22 Schoepfer A, Straumann A, Panczak R, et al: Development and validation of a symptombased activity index for adults with eosinophilic esophagitis. Gastroenterology 2014; 147:1255-1266.e21.

23 Dellon ES, Irani AM, Hill MR, Hirano I: Development and field-testing of a novel patient-reported outcome measure of dysphagia in patients with eosinophilic esophagitis. Aliment Pharmacol Ther 2013;38:634-642.

24 Patrick DL, Burke LB, Powers JH, et al: Patient-reported outcomes to support medical product labeling claims: FDA perspective. Value Health 2007;10(suppl 2):S125-S137.

25 Hirano I, Moy N, Heckman MG, et al: Endoscopic assessment of the oesophageal features of eosinophilic oesophagitis: validation of a novel classification and grading system. Gut 2013;62:489-495.

26 Collins MH, Martin LJ, Alexander ES, et al: Newly developed and validated eosinophilic esophagitis histology scoring system and evidence that it outperforms peak eosinophil count for disease diagnosis and monitoring. Dis Esophagus 2017;30:1-8.

27 Hruz P, Straumann A, Bussmann C, et al: Escalating incidence and prevalence of eosinophilic esophagitis: a 20-year prospective, population-based study in Olten County, Switzerland. J Allergy Clin Immunol 2011;128: 1349-1350.

28 Giriens B, Yan P, Safroneeva E, et al: Escalating incidence and prevalence of eosinophilic esophagitis in Canton of Vaud, Switzerland, 1993-2013: a population-based study. Allergy 2015;70:1633-1639.

29 Pittet V, Juillerat P, Mottet C, et al: Cohort profile: the Swiss Inflammatory Bowel Disease Cohort Study (SIBDCS). Int J Epidemiol 2009;38:922-931.

30 Molina-Infante J, Schoepfer AM, Lucendo AJ, Dellon ES: Eosinophilic esophagitis: what can we learn from Crohn's disease? United European Gastroenterol J 2017;5:762-772.

31 Safroneeva E, Straumann A, Coslovsky M, et al: Symptoms have modest accuracy in detecting endoscopic and histologic remission in adults with eosinophilic esophagitis. Gastroenterology 2016;150:581-590. 